presence of DCI are both associated with increased mRS at 90 days.

Conclusions Use of ASA does not appear to confer worse outcomes in cerebral aneurysm rupture and subarachnoid hemorrhage.

Disclosures V. Patel: None. F. Al Mufti: None. R. Gupta: None.

\section{E-148 DECOMPRESSIVE CRANIECTOMY IN ACUTE STROKE PATIENTS IN KOREAN SINGLE CENTER}

K Jang*, B Cho, B Moon, K Kim, D Jang. Neurosurgery, Incheon St. Mary's hospital, The Catholic University of Korea, Bupyeong-gu, Incheon, Korea, republic of

\subsection{6/neurintsurg-2019-SNIS.223}

Introduction Decompressive craniectomy (DC) is a life-saving surgical procedure being increasingly employed for malignant intracerebral artery stroke. The purpose of this study is to investigate the characteristics and prognostic factors of patients undergoing DC after acute ischemic stroke.

Method From 2013 to 2018, we analyzed the prospective gathered data of 58 consecutive patients treated with DC after acute ischemic stroke in a single center. Also, we analyzed the prospective gathered data of 160 consecutive patients treated with mechanical thrombectomy using stent-retrievers for acute ischemic stroke in a single center. Demographic, clinical, laboratory, and radiologic features of each patient were investigated. We compared the characteristics of patients who underwent DC with those of patients who received medical treatment.

Results Of the total 3884 acute ischemic stroke patients, 58 patients(1.49\%) were underwent DC. Of 289 patients who underwent mechanical thrombectomy due to acute ischemic stroke, DC was performed in 32 patients(11.07\%). Of the patients who underwent mechanical thrombectomy due to acute ischemic stroke, post thrombectomy complications $(100 \%$ vs $22.81 \%, p=0.000)$, preprocedural TICI grade $(p=0.001)$ were significantly higher in patients who underwent DC compared to patients who did not receive craniectomy. 30 days mRS scores(5.13 vs 3.37, p=0.000), 90 days mRS scores(5.06 vs $3.17, \mathrm{p}=0.000)$, ICU hospital days $(21.53$ vs $8.65, \mathrm{p}=0.000)$ and hospital mortality $(46.88 \%$ vs $12.5 \%$, $\mathrm{p}=0.000)$ were significantly higher in the craniectomy patients.

Conclusions Patients who underwent DC had more post procedural complications, and the functional outcomes were worse after DC in acute ischemic stroke. However, since DC is the last treatment option for patients with severe cerebral infarction who are not responding to medical treatment, it is advisable to consider active management while managing the underlying disease and periprocedural complications.

Disclosures K. Jang: None. B. Cho: None. B. Moon: None. K. Kim: None. D. Jang: None.

\section{Late-Breaking Oral Abstracts}

\section{THE RIVER TRIAL: A TRIAL OF VENOUS SINUS STENTING WITH THE RIVER STENT IN IDIOPATHIC INTRACRANIAL HYPERTENSION (IIH)}

A Patsalides*. Weill Cornell Medical College, New York, NY

\subsection{6/neurintsurg-2019-SNIS.224}

Introduction Venous sinus Stenting (VSS) is an emerging treatment for patients with IIH. However, VSS is currently performed with stents designed for stenting atherosclerotic arterial stenosis. The River ${ }^{\mathrm{TM}}$ stent has been specifically designed for treating the stenosis of the transverse-sigmoid sinuses and has the following characteristics: easy navigability through the tortuosity of the sigmoid sinus; $7 \mathrm{~cm}$ long length to cover the venous sinuses from the torcula to the mid sigmoid segment; two zones of radial force to address the intrinsic stenosis at the sigmoid transverse junction (stiffer zone) and extrinsic stenosis of the mid transverse sinus (softer zone); and large open cells to adapt to the triangular shape of the venous sinus without occluding cortical veins.

Methods The River trial is a single arm pivotal trial designed for HDE approval of the River ${ }^{\mathrm{TM}}$ stent (Serenity Medical Inc, Redwood City, CA). The trial will enroll 39 subjects in 7 clinical sites. Subjects must have proven $\mathrm{IIH}$ and $>50 \%$ stenosis of the sigmoid-transverse sinus with pressure gradient $\geq 8 \mathrm{~mm}$ $\mathrm{Hg}$. Subjects can be enrolled on visual criteria: visual field deficit from $-6 \mathrm{db}$ to $-30 \mathrm{db}$ resistant to medical therapy $>2$ weeks; or on headaches criteria: severe headaches defined by Headache Impact Test (HIT-6) $>59$ resistant to medical therapy $>4$ weeks. The patients need to be on dual antiplatelet therapy (aspirin and clopidogrel) for 3 months and aspirin monotherapy for 9 more months.

The primary safety endpoint is the rate of major adverse event (any neurological complication plus need for any surgical procedure for $\mathrm{IIH}$ ) at 12 months. The primary efficacy endpoint is a composite at 12 months of clinical improvement and absence of significant venous stenosis. Secondary endpoints at 12 months include opening pressure on lumbar puncture, individual ophthalmology endpoints, change in pulsatile tinnitus, change in medications, change in quality of life scores.

Results Enrollment started in September 2018. Four sites are active at the time of submission of this abstract and three other sites are in the process of certification. Eleven subjects have been enrolled. In all 11 cases the catheterization with the River $^{\mathrm{TM}}$ stent was extremely easy and the stent was deployed as expected. In two patients with severe stenosis from arachnoid granulation, post-stent angioplasty was performed. There was resolution of the trans-stenotic gradient in all cases. There was no stent-thrombosis. There was no impairment of flow in the cortical veins draining into the stented segment. There were no major neurological adverse events or device related complications.

None of the enrolled patients reached the 12-month follow-up time point and therefore we cannot report on primary 
and secondary endpoints but an update of the trial with intermediate clinical results will be presented at the conference. Conclusion We provide early/interim results of the use of the River stent. This novel stent has been effective for the treatment of venous sinus stenosis without any short-term serious adverse events or device related complications.

Disclosures A. Patsalides: None.

\section{IMAGING TRIAGE OF LATE WINDOW PATIENTS WITH ACUTE ISCHEMIC STROKE. A COMPARATIVE STUDY USING MULTI-PHASE CT ANGIOGRAPHY VS CT PERFUSION}

${ }^{1} \mathrm{M}$ Almekhlafi ${ }^{*},{ }^{2} \mathrm{~W}$ Kunz, ${ }^{3} \mathrm{R}$ McTaggart, ${ }^{4} \mathrm{M}$ Jayaraman, ${ }^{1} \mathrm{M}$ Najm, ${ }^{5} \mathrm{~S}$ Ahn, ${ }^{6} \mathrm{E}$ Fainardi, ${ }^{7} \mathrm{M}$ Rubiera, ${ }^{8} \mathrm{~A}$ Khaw, ${ }^{9} \mathrm{~A}$ Zini, ${ }^{10} \mathrm{M}$ Hill, ${ }^{10} \mathrm{~A}$ Demchuk, ${ }^{10} \mathrm{M}$ Goyal, ${ }^{10} \mathrm{~B}$ Menon. ${ }^{1}$ University of Calgary, Calgary, AB, Canada; ' $U$ niversity of Calgary, Munich, AB, Canada; ${ }^{3}$ Warren Alpert Medical School of Brown University, Providence, $R I_{;}{ }^{4}$ Warren Alpert Medical School of Brown University, Calgary, AB, Rl; ${ }^{5}$ Chosun University School of Medicine, Gwang Ju, Korea, Republic of; ${ }^{6}$ University Hospital, Ferrara, Ferrara, Italy; ${ }^{7}$ Hospital Vall d'Hebron, Barcelona, Spain; ${ }^{8}$ University of Western Ontario, London, ON, Canada; ${ }^{9}$ Maggiore Hospital, Bologna, Italy; ${ }^{10}$ University of Calgary, Calgary, AB, Canada

\subsection{6/neurintsurg-2019-SNIS.225}

Background Current guidelines recommend the use of perfusion imaging for selection of patients for endovascular thrombectomy (EVT) beyond six hours from onset. The role of collateral imaging in this time window is not established.

Methods We used data from a prospective multi-center observational study where all stroke patients with suspected large vessel occlusion underwent imaging with single- and multiphase CT angiography (mCTA) as well as CT perfusion. For this analysis, we only included patients presenting beyond six hours from onset/last known well time. Two blinded reviewers judged patients' eligibility for EVT using published collateral imaging (mCTA), compared to CT perfusion (using DAWN and DEFUSE-3 trials) selection criteria. All perfusion images were processed using an automated commercial software. The outcomes of patients eligible for EVT using mCTA, DAWN, or DEFUSE-3 criteria were compared using multivariable logistic regression modeling. Model predictive characteristics were assessed using c-statistic for the receiver operating curve, Akaike information criterion (AIC), and Bayesian information criterion (BIC).

Results Of 614 patients, 86 patients presented beyond six hours from onset/last known well (median 9.6 hours, IQR 4.1 hours). Median age was 71 years (IQR 14 years), 48.8\% were females, median baseline NIHSS was $12(\mathrm{IQR}=11)$. Thirty-five patients $(40.7 \%)$ received EVT of which good functional outcome (90 day modified Rankin scale 0-2) was achieved in $47 \%$. Collateral-based imaging paradigms significantly modified the treatment effect of EVT on clinical outcome i.e. 90-day mRS 0-2 (P interaction $=0.007)$. The mCTA-based regression model best fit the data for 90-day outcome (C statistic 0.86, 95\% CI 0.77 to 0.94 ) and was associated with least information loss (AIC 95.7, BIC 114.9) when compared to CTP based models. Perfusion imaging paradigm using DEFUSE-3 criteria had better predictive properties than the DAWN trial criteria.

Conclusion Collateral-based imaging paradigm using mCTA compares well with CTP in selecting patients for EVT in the late time window.

Disclosures M. Almekhlafi: None. W. Kunz: None. R. McTaggart: None. M. Jayaraman: None. M. Najm: None. S. Ahn:
None. E. Fainardi: None. M. Rubiera: None. A. Khaw: None. A. Zini: None. M. Hill: None. A. Demchuk: None. M. Goyal: None. B. Menon: None.

\section{BUILDING EFFECTIVE STROKE SYSTEMS OF CARE THROUGH A REGIONAL EMERGENCY MEDICAL SERVICES (EMS) COALITION AND DATA TRANSPARENCY INITIATIVE: THE SOUTH FLORIDA EXPERIENCE}

${ }^{1} \mathrm{~B}$ Mehta* ${ }^{2} \mathrm{P}$ Antewy, ${ }^{2} \mathrm{R}$ Katz ${ }^{3} \mathrm{~J}$ Sessa, ${ }^{4} \mathrm{~K}$ Scheppke ${ }^{5} \mathrm{P}$ Pepe, ${ }^{6} \mathrm{R}$ Hanel, ${ }^{7} \mathrm{M}$ Mokin, ${ }^{8} \mathrm{C}$ Gutierrez, ${ }^{9} \mathrm{~J}$ Romano, ${ }^{10} \mathrm{R}$ Nogueira, ${ }^{11} \mathrm{~A}$ Jadhav, ${ }^{12} \mathrm{~T}$ Leslie-Mazwi, ${ }^{9} \mathrm{R}$ Sacco. ${ }^{1}$ Neurosurgery, Memorial Healthcare System, Hollywood, FL; ${ }^{2}$ Emergency Medicine, Memorial Healthcare System, Hollywood, FL; ${ }^{3}$ Neurology, Memorial Healthcare System, Hollywood, FL; ${ }^{4}$ Palm Beach County Fire Rescue, West Palm Beach, FL; ${ }^{5}$ Emergency Medicine, UT Southwestern Medical Center, Dallas, TX; ${ }^{6}$ Neurosurgery, Baptist Health, Jacksonville, FL; ${ }^{7}$ Neurosurgery, USF Health, Tampa, FL; ${ }^{8}$ Neurology, University of Miami, Miami, FL; ${ }^{9}$ Neurology, University of Miami School of Medicine, Miami, FL; ${ }^{10}$ Neurosurgery, Emory University School of Medicine, Atlanta, GA; ${ }^{11}$ Neurology, University of Pittsburgh Medical Center, Pittsburgh, PA; ${ }^{12}$ Neurosurgery, Massachusetts General Hospital, Boston, $M A$

\subsection{6/neurintsurg-2019-SNIS.226}

Introduction Advances in acute stroke treatment of emergency large vessel occlusions (ELVO) have led to a transformation in the delivery of stroke care. Because of the importance of time to treatment with endovascular thrombectomy (EVT), there has been renewed emphasis on improving stroke systems of care. In south Florida, there has been a proliferation of stroke centers over the past decade claiming to offer EVT due to the practice of self-attestation by the Agency for Healthcare Administration (AHCA). Concurrently, there are over a dozen EMS agencies in the region, leading to variability in pre-hospital protocols.

Methods To address the fragmented stroke landscape, in 2015 local EMS Medical Directors along with NeuroInterventionalists (NI) developed the South Florida Stroke Coalition (SFSC), and collaborated with the University of Miami Florida Stroke Registry (UM FSR) team. The core tenets were to: 1) Develop pre-hospital EMS stroke protocols; 2) Optimize in-hospital stroke processes to reduce times to treatment for ELVO; 3) Encourage all tri-county stroke centers to submit data to AHA/ASA Get with the Guidelines-Stroke (GWTG-S) program and participate in the UM FSR; 4) Develop a sustainable data transparency model for CSCs to allow EMS Medical Directors to understand quality of stroke care in their region; 5) Eliminate the practice of AHCA self-attestation of CSCs and require stroke centers to apply for Joint Commission certification.

Results The SFSC and the UM FSR analyzed AHCA criteria for Comprehensive Stroke Center (CSC) designation and compared them to those of the Joint Commission to better understand limitations of self-attestation. A sustainable mechanism for local hospitals to share stroke data with the EMS Medical Directors in their catchment areas was created through a regional dashboard initiative (figure 1). The following key variables were selected by the SFSC to provide a better understanding of the quality of care: 1) treatment rates for tPA and EVT, 2) door-to-needle time for tPA, 3) door-to-puncture time for EVT, 4) outcomes at 90 days using modified Rankin scale. Conclusion To our knowledge, the Florida Stroke Registry Regional Dashboards developed as a grassroots effort between the SFSC and the UM FSR represents the first-of-its-kind, EMS-driven effort to improve stroke systems of care in a 